Meta

Journal des tradlucteurs

Translators' Journal

\title{
Dubuc, Robert (1996) : Une grammaire pour écrire. Essai de grammaire stylistique, Montréal, Linguatech, 262 p.
}

\section{Hélène Cajolet-Laganière}

Volume 42, numéro 4, décembre 1997

URI : https://id.erudit.org/iderudit/003375ar

DOI : https://doi.org/10.7202/003375ar

Aller au sommaire du numéro

\section{Éditeur(s)}

Les Presses de l'Université de Montréal

\section{ISSN}

0026-0452 (imprimé)

1492-1421 (numérique)

Découvrir la revue

Citer ce compte rendu

Cajolet-Laganière, H. (1997). Compte rendu de [Dubuc, Robert (1996) : Une grammaire pour écrire. Essai de grammaire stylistique, Montréal, Linguatech, 262 p.] Meta, 42(4), 726-729. https://doi.org/10.7202/003375ar

Ce document est protégé par la loi sur le droit d'auteur. L'utilisation des services d’Érudit (y compris la reproduction) est assujettie à sa politique d'utilisation que vous pouvez consulter en ligne.

https://apropos.erudit.org/fr/usagers/politique-dutilisation/ 
DUBUC, Robert (1996): Une grammaire pour écrire. Essai de grammaire stylistique, Montréal, Linguatech, 262 p.

J'ai lu avec beaucoup d'intérêt et de plaisir, je devrais plutôt dire que j'ai dégusté le livre de Robert Dubuc Une grammaire pour écrire, essai de grammaire stylistique. Cet ouvrage apparaît comme un bain de fraîcheur à travers les nombreuses grammaires et dictionnaires de difficultés du français, dont les règles semblent souvent arides et difficilement applicables aux lecteurs et lectrices. Cet essai de grammaire stylistique arrive à point, au moment où, au Québec, on se préoccupe de norme et d'usages propres au français écrit tel qu'il est utilisé dans les textes soignés des Québécois et Québécoises. L'ouvrage est une incitation à l'écriture. De fait, l'auteur nous invite à nous approprier la langue écrite, à mettre de côté la crainte de l'erreur et de la critique et à le suivre dans ce guide visant à mettre en évidence les richesses du code écrit afin de clarifier, d'enrichir et de nuancer l'expression de la pensée.

Selon l'auteur, ce livre s'adresse à ceux et celles qui écrivent par besoin, par passion ou par profession : écrivains, novices ou vétérans, rédacteurs de toute farine, y compris la 
publicitaire, traducteurs techniques ou littéraires. Tous et toutes y trouveront de nouvelles ressources expressives à exploiter et un intérêt nouveau pour une grammaire devenue servante plutôt que bourreau.

L'ouvrage se divise en deux parties. Dans un premier temps, l'auteur passe en revue les principales parties du discours et l'articulation logique des énoncés. La deuxième partie est consacrée à une série d'exercices visant à l'assimilation de la matière exposée dans les huit chapitres de l'ouvrage. Plusieurs qualités caractérisent cet essai: un style vivant, simple et clair; une manière très personnelle et originale de présenter les ressources stylistiques qu'offre la grammaire; une matière riche et dense tirée de l'expérience de l'auteur, qu'il nous présente avec générosité, finesse et intelligence; des faits langagiers d'une grande actualité provenant de différents types de discours et registres de langue (oral, littéraire, technique, journalistique, publicitaire et autres), le tout agrémenté d'exemples qui illustrent chaque affirmation; des conseils enfin pour respecter le caractère idiomatique du français, qui, au Québec, est constamment en contact avec l'anglais.

Les deux premiers chapitres sont particulièrement intéressants et novateurs. Le premier, «L'article ou l'essence d'un mot-outil», présente en détail les diverses fonctions de l'article dans la phrase française. Comme le souligne l'auteur, nous utilisons l'article par instinct, et le plus souvent cet instinct est bien inspiré. Mais il y a toujours des zones d'ombre où l'instinct n'arrive pas avec sûreté à trouver la construction qui convient ou à identifier les raisons pour lesquelles un énoncé apparaît boîteux. C'est pourquoi, ajoute l'auteur, il importe de découvrir les mécanismes qui sous-tendent l'emploi de l'article de façon à raisonner ce qu'on fait par instinct pour ensuite dissiper les zones d'ombre en toute connaissance de cause. C'est ainsi qu'à travers ce chapitre, l'auteur passe en revue les différents emplois stylistiques de l'article vu comme un outil de mise en valeur: valeur de présentation et de caractérisation, valeur distributive et valeur emphatique. Grâce à de nombreux exemples très pertinents et actuels, il nous apprend à connaître et à exploiter les différents procédés et ressources stylistiques de l'article selon les différents registres de langue (populaire, soutenu, littéraire).

L'auteur fait état de toutes les possibilités qu'offre l'article avec les nuances qu'elles comportent. Beaucoup d'aspects traités dans ce chapitre, notamment les explications et les exemples fournis par l'auteur, sont difficilement accessibles dans les différents ouvrages de référence d'usage courant. Cela contribue à l'intérêt et à la richesse de ce guide. En outre, l'auteur prend soin de distinguer ce qui est régi par les règles grammaticales et, par ailleurs, les cas d'arbitraire, les caprices de la langue, tout en faisant une analyse fine des nuances stylistiques amenées par ces différents emplois.

Le deuxième chapitre, consacré à «L'univers de l'adjectif qualificatif», est tout aussi intéressant et novateur quant au traitement de l'information. Pour l'auteur, le juste maniement de l'adjectif est la marque d'un bon styliste. Aussi celui-ci tente-t-il de rendre la rédactrice ou le rédacteur conscient des différences de sens ou d'effets obtenus en déplaçant l'adjectif, en le transvaluant par rapport à un complément déterminatif ou à un adverbe, dans le but de lui permettre de tirer un bon parti des ressources stylistiques de l'adjectif. En ce sens, il décrit les diverses modes qui ont influé sur l'utilisation de l'adjectif à travers les âges; il explicite par la suite les emplois actuels de l'adjectif dans la langue courante, littéraire et technique et les interférences avec la langue anglaise et les principaux procédés qui ont l'adjectif comme pivot: le déplacement, la transvaluation et l'hypallage. Cela engendre divers effets affectifs et donne à la phrase un relief, une force d'évocation (effet de surprise et d'inattendu, d'ironie, etc.).

Pour l'auteur, l'analyse du sens de l'énoncé constitue la clé du maniement stylistique de l'adjectif. Même pour l'accord, le sens joue un rôle capital et justifie les pratiques qui pourraient autrement sembler purement arbitraires. 
Le troisième chapitre, intitulé «Le nom, appellation ou désignation», insiste sur l'importance du genre et du nombre dans toute l'étude du substantif, car ces derniers peuvent aussi avoir une incidence sémantique. Il rappelle et démontre avec justesse que le sens des noms est en constante évolution sous la poussée des besoins d'expression réels et sentis et que les mutations de sens ne sont pas pour autant arbitraires. En ce sens, l'auteur aborde les particularités propres aux noms propres, aux noms communs simples et composés, aux syntagmes lexicalisés ou en voie de l'être. Il traite des problèmes relatifs au genre et au nombre des noms, à la féminisation, à l'orthographe d'usage et à l'accord des noms. Il y va même de certaines propositions propres à réformer l'orthographe d'accord dans le but d'éviter l'anarchie qui règne parfois dans le secteur du nombre.

Dans le chapitre quatre, «Les traquenards des pronoms», l'accent est mis sur l'importance des rapports qui unissent le pronom à son antécédent. Le rédacteur doit toujours veiller à associer pronom et antécédent sans aucune ambiguïté. Aussi, l'auteur prend soin de mettre en garde le rédacteur face à un certain nombre d'erreurs types concernant notamment l'emploi des pronoms interrogatifs «qui», «que», «quoi», du pronom indéfini «on», du pronom relatif «dont», des pronoms adverbiaux «en» et «y», etc.

Par ailleurs, l'accent est également mis sur les emplois stylistiques des pronoms, soit pour marquer l'insistance, la politesse, entre autres, l'emploi approprié des pronoms de modestie «nous» et «vous», la tendresse ou l'affection, ou encore une nuance d'imprécision. Ici encore, les liens sont faits d'une manière précise et fine avec les différents types de discours (langue administrative, littéraire, juridique, de spécialité et autres). L'auteur note enfin certaines inélégances de l'expression à éviter quant à l'emploi de certains pronoms ainsi que la lourdeur des relatifs composés «lequel», «laquelle», etc.

Les chapitres cinq, six et sept traitent du verbe en tant que moteur de la phrase : ils exposent en outre les modes et usages du verbe et abordent l'épineuse question de la concordance des temps en français moderne et actuel. L'auteur fait état des distinctions entre langue parlée et langue écrite, en pointant certaines particularités des discours techniques et scientifiques. Il explicite en outre les valeurs stylistiques et affectives propres aux différents modes, temps et voix: l'affection, l'atténuation, l'hypothèse, etc. Ces chapitres constituent un guide très sûr quant à une utilisation juste et appropriée des temps et modes; il permet en outre au rédacteur de développer un sens des nuances adapté aux divers discours et types de communication (langue journalistique, technique et scientifique).

L'auteur fait de plus ressortir l'importance du sens de l'énoncé; c'est le plus souvent lui qui dicte l'accord et les temps et modes à employer. Fort de son expérience, l'auteur réagit enfin contre certaines tendances actuelles qui faussent le sens des modes. Selon ce dernier, il importe de connaître les ressources de la langue, et au besoin, forcer les servitudes grammaticales si, par cette espèce de violence faite aux habitudes du langage, on parvient à exprimer ce qu'il ne serait pas possible d'exprimer autrement.

Enfin, le huitième et dernier chapitre est consacré à «L'articulation de l'énoncé». Ce chapitre traite de trois éléments essentiels à la compréhension du texte par le destinataire visé, soit la structuration de l'énoncé (idées principales et secondaires), les paragraphes, les charnières du discours, tant implicites qu'explicites, qui sont garantes de la cohésion du texte par les liens entre les idées et la ponctuation, qui assure la bonne articulation du texte. Elle permet de délimiter les unités syntaxiques, de mettre en relief certains éléments de l'énoncé et de clarifier, au besoin, les unités de discours à coordonner. Toutefois, pour l'auteur, écrire n'est pas uniquement une question de style. C'est aussi un acte de communication, qui ne peut atteindre pleinement son but que dans une fidélité éclairée au code grammatical. 
L'ouvrage se termine par une série d'exercices originaux et fort bien pensés, permettant au lecteur ou à la lectrice de mieux comprendre et d'assimiler les notions présentées dans les huit chapitres de l'ouvrage. Une courte bibliographie et un index complètent l'ouvrage.

Au terme de ce bref compte rendu, il me reste à remercier l'auteur, Robert Dubuc, d'avoir mis à notre disposition cet Essai de grammaire stylistique, fruit de sa longue et riche expérience en tant que rédacteur, réviseur, traducteur et conseiller linguistique. En parcourant cet ouvrage, le lecteur ou la lectrice aura accès à une synthèse de l'auteur des éléments stylistiques du code grammatical. Robert Dubuc nous offre en outre une prise de conscience des ressources que nous pouvons y puiser. Il nous invite enfin à enrichir notre palette expressive et à mieux la maîtriser.

HÉLÈnE CAJOLET-LAGANIÈRE Université de Sherbrooke, Sherbrooke, Canada 